\title{
TRES MODELOS DE ANÁLISIS DEL SIGNFICADO. APROXIMACIÓN METODOLÓGICA AL ESTUDIO DE LAS EMOCIONES
}

\author{
Carmelo Pérez Rull. I.E.S. Al-Ándalus, Almería
}

\begin{abstract}
Este artículo se divide en dos secciones principales. La primera está dedicada a una breve descripción teórica de tres modelos de análisis del significado: el modelo intensional, el modelo de Wierzbicka y el modelo cognitivo-cultural. El lenguaje en gran medida refleja la experiencia humana y tiene sentido cuando se le analiza desde un punto de vista cognitivo-cultural. Por esta razón, en la segunda parte clarificamos nuestra propuesta metodológica del estudio de las emociones según el modelo de causa-efecto, el modelo analítico y el modelo sintético. En el nivel de causa-efecto representamos los elementos del evento emocional. En la fase analítica partimos con los esquemas cognitivos culturales subyacentes a una predicación dada o corpus lingüístico. Con la orientación sintética comenzamos con el nivel de conceptualización pragmático cognitivo, después estudiamos el nivel de conceptualización simbólica $\mathrm{y}$, finalmente, se termina con las codificaciones lingüísticas.
\end{abstract}

\section{INTRODUCCIÓN}

Las emociones son procesos complejos formados por componentes innatos, subjetivos, fisiológicos y conductuales. Su estudio debe ser globalizador y no debe quedar reducido a cada uno de sus componentes. El acercamiento al estudio emocionológico debería incorporar información interdisciplinar acerca de su naturaleza y funciones (Martín Morillas, en prensa).

El objetivo de este trabajo es presentar una propuesta de análisis del significado más allá de un acercamiento con criterios lingüísticos al estudio de las emociones. Es necesario desvelar la estructura psicológica subyacente de las emociones a partir de los datos aportados por los individuos, como resultado de sus experiencias particulares en relación a determinadas emociones. Deberíamos considerar tanto el conocimiento lingüístico como el extralingüístico, ya que expresamos intenciones comunicativas que son proyecciones de determinados modelos culturales que representan y codifican todo lo que saben los miembros de una determinada cultura: creencias, valores y actitudes.

Hemos comenzado este trabajo con una breve descripción de tres modelos de análisis del significado que pensamos que se deberían tener en cuenta: el modelo intensional, el modelo de Wierzbicka y el modelo cognitivo-cultural y, a continuación, presentamos nuestra propuesta del 
estudio metodológico de las emociones: modelo de causa-efecto, modelo analítico y modelo sintético.

\section{EL MODELO INTENSIONAL}

Para los estructuralistas clásicos (Coseriu, 1986; Geckeler, 1984), el significado de una unidad léxica depende del análisis del significado de las otras unidades léxicas del campo léxico, dejando a un lado las relaciones psicolingüísticas y gnoseológicas entre el conocimiento lingüístico y extralingüístico, o el papel de la mente humana en la creación psico-motivacional de los significados. Se centra en las relaciones de sentido y en la organización interna que crean los conjuntos léxicos.

La principal aportación que hace Coseriu a la semántica estructural consiste en estudiar el componente léxico estructurado en sistemas articulatorios funcionalmente. Para él, el objeto de la lexemática son las relaciones de significación, distinguiendo lo que es pertinente o no pertinente a la estructura del significado. Por otro lado, la lexemática se preocupa también de las relaciones estructurales paradigmáticas y sintagmáticas de los significados léxicos dentro de un mismo sistema lingüístico.

Recientemente, la escuela lexemática funcional (Martín Mingorance, 1998) ha buscado superar estas limitaciones, incorporando el nivel cognitivo dentro del sistema de definición léxica. Las bases teóricas de este modelo proceden tanto de la Gramática Funcional de S. C. Dik (1978) como de la lexemática desarrollada por Coseriu y Geckeler. El enfoque LF considera la lengua como un sistema de comunicación que se desenvuelve en el plano semántico, sintáctico y pragmático. Los lexemas se definen dentro de redes de campos léxicos, mediante la descomposición léxica hiperonímica, dentro de la propia lengua funcional, según el llamado principio de la descomposición léxica gradual.

\section{EL MODELO DE WIERZBICKA}

Según este modelo cada lengua impone su propia clasificación de los conceptos emocionales basándose en la experiencia humana y, por lo tanto, cualquier palabra emocional, por ejemplo "sadness" no está exenta del componente cultural. Una forma de describir el significado de las emociones, sin caer en la circularidad, es recurrir a los primitivos semánticos o conceptos universales tales como: 'want', 'feel', 'think', 'say', 'good' o 'bad'. La serie de primitivos semánticos universales propuestos por Wierzbicka (1996: cap 3) se ha extendido desde 14 en 1972 hasta 55 en $1996^{1}$. Estos términos no son artefactos culturales de la lengua inglesa, sino que pertenecen al alfabeto universal del pensamiento humano.

El objetivo del modelo de Wierzbicka está en encontrar los conceptos humanos universales con el fin de analizar el significado de las palabras a través de primitivos semánticos, ya que los sistemas semánticos de las diferentes lenguas son específicos de cada cultura. A través de la descomposición de los significados de las palabras en primitivos semánticos es como podemos investigar de forma sistematizada y metodológica la estructura de los campos semánticos. Una vez que hayamos identificado la estructura interna del significado de cada palabra, podemos desvelar también las relaciones estructurales que existen entre las palabras (Wierzbicka, 1996: cap 5)

\footnotetext{
${ }^{1}$ En 1998 el número de primitivos semánticos se reducen a 54, la contrafactualidad fue borrada de la lista en un taller sobre NSM celebrado en la Universidad La Trobe de Australia, sobre la base de datos del chino mandarín.
}

Odisea, $\mathrm{n}^{0} 1,2001$ 
El modelo semántico de Wierzbicka define los conceptos de emoción por medio de prototipos y guiones. Propone un análisis conceptual preciso que nos permite captar los aspectos más sutiles y elusivos del significado. De esta forma, la estructura semántica de la mayoría de los conceptos se podrían representar de acuerdo con un guión:

Analicemos el siguiente concepto de emoción: DEPRESSED

\section{Estructura conceptual}

$\mathrm{X}$ feels something

sometimes a person thinks something like this:

I can think: something bad will happen to me

I can't think: something good will happen to me

I can't think: I will do something good

because of this, this person feels something bad

$\mathrm{X}$ feels something like this

El modelo de Wierzbicka, a pesar de encontrarse en fase de investigación el número de primitivos semánticos, puede incorporarse al estudio emocionológico ya que un término o expresión emotiva puede ser captado mediante una representación semántica con un formato de un guión de los escenarios prototípicos de la expresión emotiva.

\section{EL MODELO COGNITIVO-CULTURAL}

No existe un enfoque cognitivo unitario, sino una gran variedad de enfoques o paradigmas que tienen en común el énfasis en los fenómenos mentales como agentes del comportamiento. El objetivo es conocer cómo el ser humano adquiere la información, cómo la representa, la transforma y la recupera una vez almacenada, para luego actuar en el mundo de forma significativa.

El paradigma cognitivista mantiene que nuestras capacidades simbólicas están íntimamente enraizadas en la experiencia. Las estructuras conceptuales: esquemas, guiones, marcos, modelos cognitivos, etc., se establecen a través de la reiterada interacción del individuo con su entorno y dependen no tanto de una supuesta estructura inherente al mundo sino de nuestra forma de percibir, manipular e interactuar con nuestro entorno físico y social.

\subsection{Categorías y conceptos}

El pensamiento humano utiliza los conceptos y categorías como forma de organizar el conocimiento. Un concepto es una representación mental de un conjunto de objetos o hechos. Una categoría es una clase de objetos o de hechos que tienden a agruparse juntos. Al formar categorías no reflejamos necesariamente la estructura existente en el mundo sino que más bien reflejamos esa estructura tal como la percibimos (Lakoff, 1987). Las categorías se organizan jerárquicamente en tres niveles: categorías de nivel básico: coche, categorías supraordinadas: vehículo y subordinadas: coche deportivo. Un ejemplo de las categorías de emoción referido a esta taxonomía sería anger que es un término de emoción más básico que annoyance or emotion. Al pertenecer anger a la categoría de nivel básico se encontraría entre la categoría de nivel supraordinado emotion y la categoría de nivel subordinado de annoyance. El nivel básico de las categorías desempeña el papel central en el sistema cognitivo ya que sus elementos corresponden a los objetos que constituyen nuestra experiencia cotidiana y muestran la estructura correlacional del mundo, tal como la percibimos. 
Nuestro sistema conceptual se fundamenta en los conceptos de nivel básico y en los modelos de esquema de imágenes ${ }^{2}$. Entendemos estos conceptos de forma directa y automática. Los restantes conceptos que aún necesitamos para darle sentido a nuestra experiencia se desarrollan a partir de las proyecciones metafóricas, mediante los modelos cognitivos, tales como los modelos proposicionales, los modelos imagísticos, los modelos metafóricos y los modelos metonímicos.

\subsection{Las extensiones de las categorías: la metonimia y la metáfora}

Nuestra capacidad cognitiva nos permite extender los significados literales de las categorías y usarlas con un sentido no literal. La metáfora cognitiva no es un recurso estilístico, sino un proceso mental por el que estructuramos un concepto a partir de otro (Lakoff and Johnson, 1980) Las metáforas conceptuales sirven para comprender los dominios abstractos de la experiencia, como son las emociones, al relacionar o aparear tales dominios con otros dominios más concretos de nuestra experiencia física. Una expresión metafórica es una manifestación de una metáfora conceptual. Boiling with anger es un ejemplo lingüístico de la metáfora conceptual ANGER IS A HOT FLUID. Los conceptos metafóricos no son arbitrarios sino que se basan en nuestra experiencia física y cultural. Un gran número de nuestros conceptos están organizados en términos de metáforas orientativas: HAPPINESS IS UP eg. I am feeling high, he is walking on air; SADNESS IS DOWN eg. I am feeling down, he hit bottom when his wife died. Los esquemas imagísticos son el fundamento de las proyecciones metafóricas y metonímicas. En estos ejemplos el esquema imagístico de la verticalidad que emerge de nuestra experiencia en relación a la fuerza de la gravedad o del movimiento ascendente o descendente se proyecta sobre las emociones de la felicidad y de la tristeza para poder hablar de ellas.

Un gran número de expresiones lingüísticas son metonimias, que a diferencia de las metáforas conceptuales, son proyecciones dentro de un mismo dominio conceptual. La expresión lingüística to have cold feet es un ejemplo de la metonimia conceptual DROP IN BODY TEMPERATURE STANDS FOR FEAR. Kövecses y Lakoff (Lakoff, 1987:382) han postulado un principio metonímico popular según el cual: "LOS EFECTOS FISIOLÓGICOS DE UNA EMOCIÓN REPRESENTAN A ESA EMOCIÓN”. La conexión existente entre las emociones y los síntomas es la base para la formación de las proyecciones metonímicas (mappings) a partir de los cuales se basan un gran número de metáforas.

El modelo cognitivo experiencial de Lakoff asume que el pensamiento es encarnado, surge a partir de la experiencia con nuestros cuerpos y también es imaginativo, capaz de emplear la metáfora y la metonimia más allá de la representación literal de la realidad. El significado no es independiente de la naturaleza y experiencia humana sino que es corpóreo, se define en términos de nuestras experiencias físicas y sociales. Este modelo incluye en la experiencia subjetiva del individuo su experiencia cultural.

El modelo cognitivo cultural es una combinación del enfoque cognitivo que relaciona el lenguaje con la experiencia subjetiva del individuo y del enfoque antropolingüístico que relaciona el lenguaje con la experiencia intersubjetiva (Holland y Quinn, 1987; D'Andrade,

\footnotetext{
${ }^{2}$ Los esquemas imagísticos son estructuras básicas y recurrentes de nuestra experiencia corporal que se proyectan metafóricamente a otras más complejas. Johnson (1987:126) distingue varios tipos de esquemas imagísticos básicos y proporciona un listado de las más generales: recipiente, fuerza, equilibrio, dirección/ trayecto, obstrucción, centro-periferia, parte-todo, contacto, ciclo, etc.
}

Odisea, $\mathrm{n}^{0} 1,2001$ 
1990; Martín Morillas, 1997)³. Para la antropología cognitiva, la cultura consiste en un conjunto de sistemas de significado con representación mental compartida intersubjetivamente. El lenguaje no sólo codifica nuestra experiencia sino que también activa la información que tenemos almacenadaatravés delos modelos mentales y modelos culturales. Un modelo mental es una estructura mental abstracta que codifica conceptos. Cuando los modelos mentales se engarzan y comparten intersubjetivamente, forman modelos culturales.

Pasamos, a continuación, a presentar nuestra propuesta metodológica del estudio emocionológico, según el modelo de causa-efecto, modelo analítico y modelo sintético (Martín Morillas \& Pérez Rull, 1998) ${ }^{4}$

\section{EL MODELO/ESTUDIO FENOMENOLÓGICO DE CAUSA Y EFECTO}

Al analizar las expresiones lingüísticas como portadoras de diferentes tipos de la experiencia emotiva, tenemos en cuenta no sólo los aspectos lógicos del significado, sino también los fenomenológicos. Para el modelo de causa y efecto nos inspiramos en el modelo emocionológico general propuesto por Buck, R. (1986), que tiene en cuenta el evento antecedente que es el estímulo externo, el cual se convierte en un estímulo interno cuando queda fisiológicamente registrado. Seguidamente el estímulo interno se registra emocionalmente y se valora de forma cognitiva, formando la propia experiencia emotiva. Al mismo tiempo existen unos efectos psicosomáticos y una respuesta dirigida a la acción de acuerdo con los patrones sociales y culturales. Consideremos los siguientes ejemplos (Pérez Rull, 1998-99)

1) She was deeply shocked by her husband's death

2) He has been restless and unhappy since he lost his job

3) It breaks my heart to see you so unhappy

En este guión general de causa-efecto intervienen:

La causa emocional (W): her husband's death, he lost his job, to see you so unhappy.

La experiencia emotiva (Y) que se codifica indirectamente en: shocked y break y de forma directa mediante el término léxico-gramatical: unhappy.

El ente que experimenta la emoción (X) se conceptualiza como un objeto recipiente sobre el que se aplica una fuerza que impacta y se rompe, o como un recipiente en estado de agitación . El locus funcional del estado emotivo que corresponde al punto de aplicación de una fuerza se codifica en la expresión my heart. La intensidad emocional, que depende de la cualidad de lo inesperado o sorprendente de la causa emocional y, también de la valoración de dicha causa, teniendo en cuenta la experiencia pasada y la experiencia emocional presente del individuo, se codifica lingüísticamente mediante el adverbio deeply.

El efecto psicosomático $(Z)$ se codifica léxicamente de forma explícita en restless. Después de la interpretación cognitiva, el cuerpo reacciona con una respuesta física que manifiesta somáticamente en una pérdida de la calma y de la serenidad.

$\mathrm{Si}$ analizamos fenomenológicamente las expresiones anteriores, observamos que la experiencia emotiva se manifiesta de forma explícita en la frase (2), pero en las otras dos existe un mapping conceptual en el que de forma implícita se manifiesta la experiencia subjetiva del

\footnotetext{
${ }^{3}$ No parece existir una verdadera diferencia entre el modelo de Lakoff y el modelo cognitivo-cultural, como no sea la más sistemática y detallada incorporación de los factores culturales.

4 Proponemos un enfoque comparativo intercultural de semántica emocionológica, en gran medida programática, ilustrando los presupuestos teóricos presentados en muestras lingüísticas del español y del inglés, relacionadas con el dominio semántico de la alegría y de la tristeza.
}

Odisea, $n^{0} 1,2001$ 
dolor. El significado de dichas oraciones está motivado por la metáfora del golpe que fragmenta (break) y del golpe que impacta (deeply shocked). La imagen metal que subyace a dichas expresiones es el esquema mental de la fuerza. La experiencia emotiva se codifica mediante una expresión figurada con dos verbos de acción. Existe una proyección conceptual de un golpe que impacta y fragmenta un objeto para expresar el sufrimiento de un individuo que se ve afectado por acontecimiento no deseado, que lo vivencia como un golpe.

\section{MODELO ANALÍTICO}

En este modelo estudiamos las expresiones lingüísticas como 'productos' a disposición de cada lengua e identificamos los modelos mentales: el modelo imagístico, conceptual y cultural que subyacen a las expresiones léxicas, léxico-gramaticales y léxico-discursivas. Las codificaciones lingüísticas encapsulan motivaciones cognitivas y culturales que representan de forma esquemática cómo una determinada cultura se enfrenta e interpreta los sucesos del mundo. A partir de una expresión o corpus de datos podemos analizar los siguientes tipos de representación semántica:

1) Nivel extensional/proposicional: identificamos los componentes que intervienen en la ontología del evento emotivo: los sujetos y entes participantes, las causas determinantes remotas o próximas, los efectos y consecuencias, a fin de poder anclar las expresiones emotivas referencialmente. La representación ontológica nos permite identificar los elementos focales y pertinentes que el hablante puede disponer en su formulación esquematizada de un mundo evocado en forma de proposición.

2) Nivel de representación intensionalista que estudia la lengua como un sistema estructurado de signos que se conectan entre sí, sin tener en cuenta lo extralingüístico, ie. lo que las personas piensan o sienten.

3) Nivel cognitivo experiencial que hace hincapié en la relación del lenguaje con la mente y con la propia experiencia subjetiva del individuo. La conceptualización de la experiencia es el producto de nuestros procesos mentales y corporales. El significado está motivado psicogénicamente.

4) Nivel cognitivo-cultural que enfatiza las relaciones sociales del lenguaje, de la experiencia subjetiva y las condiciones sociales que afectan la comunicación por medio del habla. Este modelo conecta lo subjetivo con lo intersubjetivo, las relaciones del significado privado con las representaciones públicas y culturales

5) El nivel lingüístico codifica y categoriza los esquemas y modelos mentales. Cuando formulamos una expresión lingüística se activa información conceptual y se movilizan recursos cognitivos. La frase sintetiza los recursos de categorización y codificación a nuestra disposición como parte de nuestro conocimiento.

A continuación, ilustramos con un ejemplo en español las diferentes formas de representación semántica subyacente a la siguiente oración:

Ejemplo: Yo no pude reprimir mis lágrimas de alegría cuando la vi

1) Modelo proposicional

W: causa: explícita: cuando la vi

$\mathrm{X}$ : ente que experimenta la emoción: yo

Y: experiencia emotiva: alegría

Z: efecto: somatización de la emoción: lágrimas

2) Modelo intensional

Odisea, $\mathrm{n}^{\circ} 1,2001$ 
La oración codifica de forma directa tanto la experiencia emotiva: alegría [felicidad: en alto grado] como el efecto fisiológico: lágrimas

3) Modelo fenomenológico

- Modelo de imagen mental: el esquema subyacente a la frase es el de la fuerza y contrafuerza

- Modelo conceptual: las emociones son enemigos contra los que luchamos

4) Modelo cultural

Las emociones tienen que ser reguladas y controladas

A través de un análisis de 'desguace' de dicha codificación lingüística, observamos que proposicionalmente la oración categoriza una causa conocida que provoca la experiencia emotiva de la persona afectada que se manifiesta fisiológicamente en lágrimas. La emoción se imagina de forma conceptual como un enemigo que hay que reprimir y mantener oculto ante los demás y, por lo tanto, la idea de fuerza provocada por la emoción y de contrafuerza por parte de la persona afectada subyace a la codificación léxica. Esta frase se enmarca dentro de la fase de pérdida de control de la emoción y está codificada con un verbo de acción, de fuerza, sobre la vivencia de la emoción que se siente como un enemigo que debemos controlar. La pérdida de control sobre la emoción conceptualizada como un enemigo se manifiesta de forma somatizada en lágrimas.

\section{MODELO SINTÉTICO}

Con este modelo abordamos las expresiones como 'procesos' de generación de significado, los itinerarios dirigidos a la formación lingüística mediante un ensamblaje de recursos cognitivos gnoseológicos y procedimentales, la ontología de la representación semántica: causas, sujetos, efectos, etc., los modelos mentales y culturales, etc. Mientras que en la descripción analítica abordamos el lenguaje como un sistema o medios de recursos formales-funcionales que los hablantes disponen para evocar mundos mentales y podemos presentar una taxonomía de modelos y esquemas a partir de un corpus; es decir, operando desde las expresiones lingüísticas a las representaciones y modelos que las subyacen. En cambio, en la fase sintética se opera en sentido inverso, a partir de unas intenciones comunicativas y de unas representaciones mentales se van ensamblando o sintetizando los distintos niveles y los diversos elementos de la ontología del evento emotivo como un proceso de construcción de significado para terminar en las codificaciones lingüísticas.

Un hablante tiene varias opciones de expresar un contenido conceptual dependiendo de la situación y del tipo de codificación que quiera darle. En la fase sintética hay una intención pragmático-comunicativa, una categorización proposicional y una codificación léxica, léxicogramatical y discursiva según que las palabras, frases $\mathrm{u}$ oraciones denoten directa $\mathrm{o}$ indirectamente el contenido proposicional. A continuación vamos a representar el proceso de generación de modelos y de codificación sintética, a partir del contenido conceptual: El dolor emocional se siente como un golpe físico.

Dada una situación contextual determinada, un hablante emite una oración que conlleva la descripción de una situación personal: algo ha pasado que ha causado una reacción afectiva en una persona. Estaríamos en el nivel de conceptualización pragmático-cognitiva.

En cuanto al nivel de conceptualización simbólica, tenemos:

1. Un modelo proposicional: el hablante evoca el modelo general de causa efecto en los que representa los elementos de la ontología del evento emocional: causa (W), sujeto que experimenta la acción $(\mathrm{X})$, la vivencia de la emoción $(\mathrm{Y})$ y los efectos psicosomáticos $(\mathrm{Z})$

Odisea, $n^{0} 1,2001$ 
2. Un modelo fenomenológico: el hablante activa los siguientes modelos mentales

- Modelo general: las emociones se producen por eventos externos: por un golpe físico

- Modelo de imagen mental: el esquema mental de la fuerza de un objeto

- Modelo conceptual:

Metonimia: el dolor físico representa a la emoción

Metáfora: el dolor emocional es un golpe físico

3. Nivel de formulación lingüística:

- Codificación léxica derivada: upset

Codificación léxico-gramatical: strike sb hard, strike dumb with, throw sb off balance

Ejemplificación de codificación léxico-discursiva

The bad news trew him off balance

Her death struck him hard

The divorce upset her a lot

He was struck dumb with horror and grief

Las codificaciones reflejan los diversos itinerarios que el hablante puede tomar según se focalicen o tematicen los elementos del esquema proposicional de causa-efecto. El foco cognitivo es el golpe y los demás elementos de las ejemplificaciones forma el trasfondo cognitivo tematizado. El esquema mental de la fuerza física que golpea, desequilibra y paraliza se proyecta sobre el dominio abstracto para describir el efecto emocional como algo físico. En este nivel de codificación se pueden contrastar las diferencias interlinguísticas. Por ejemplo, en la frase struck dumb with horror and grief, la codificación lexico-gramatical conlleva una ordenación de la causa, efecto y consecuencias con un verbo del dominio semántico de "golpear" seguido de la preposición "with" que funciona como introductor del efecto emotivo. En español, ese esquema lexico-gramatical no funciona con dicha preposición sino con la preposición "de". La comparación entre el español y el inglés puede hacerse observando y registrando el grado de correspondencia entre los diversos elementos de codificación que sintetizan la formulación de los elementos de la ontología del evento emotivo.

\section{CONCLUSIONES}

Un enfoque que se dedique al análisis de la delimitación de los lexemas en rasgos y dimensiones dentro de una lengua funcional sin tener en cuenta lo extralingüístico es necesario y sirve de ayuda para orientar las pautas de los infinitos sentidos contextuales en uno u otro sentido. Sin embargo, no nos proporciona una visión global de cómo los individuos conceptualizan las emociones.

La propuesta de análisis de significado de Wierzbicka conlleva el estudio de los conceptos de emoción codificados en una lengua determinada a través de los primitivos semánticos, que constituyen el alfabeto del pensamiento humano. Mediante este análisis del significado se evita cualquier influencia que pueda ejercer la lengua de estudio, ya que se trasciende lo cultural y específico de esa lengua. Sin embargo, creemos que en estudio de las emociones se deben considerar como objeto de estudio todas las realizaciones lingüísticas de la experiencia emotiva, desde la lexía hasta las expresiones libres: unidades léxicas simples, derivadas, compuestas, figuradas, frases idiomáticas y proverbios que reflejan las proyecciones conceptuales: las metáforas y las metonimias.

El modelo cognitivo-cultural incorpora lo extralingüístico, lo cultural y nuestra experiencia corpórea en el análisis del significado. Este modelo describe la fenomenología subjetiva que posibilita anclar la red conceptual en la experiencia del cuerpo y del mundo del 
individuo y tiene en cuenta los esquemas mentales compartidos con otras personas- la fenomenología intersubjetiva- que liga la existencia de términos emotivos a las formas de pensar de una cultura -los modelos culturales-.

\section{BIBLIOGRAFÍA}

Barcelona Sánchez, A., ed. 1999. Metaphor and Metonymy at the Crossroads. A Cognitive Approach. Berlin/Amsterdam, etc.: Mouton de Gruyer.

Buck, R. 1986. "The psychology of emotion". Mind and Brain. Dialogues in Cognitive Neuroscience. Eds. J. E. Le Doux and W. Hirst. Cambridge: Cambridge University Press. 275-300.

Coseriu, E. 1986. Principios de Semántica Estructural. Madrid: Gredos.

D'Andrade, R. 1990. "Some propositions about the relation between culture and human cognition". En Stigler et al. Cultural Psychology: Essays on Comparative Human Development. Cambridge: Cambridge University Pres. 65-130.

D'Andrade, R. 1995. Developments in Cultural Anthropology. New York: Cambridge University Press.

Dik, S.C. 1978. Functional Grammar. Amsterdam: North-Holland (Traduc. española: Gramática Funcional de F. Serrano Valverde y L. Martín Mingorance. Madrid: SGEL, 1981).

Holland, D. and N. Quinn. 1987. "Culture and cognition". Cultural Models in Language and Thought. Eds. D. Holland and N. Quinn. Cambridge: Cambridge University Press. 3-43.

Johnson, M. 1987. The Body in the Mind. The Bodily Basis of Meaning, Imagination, and Reason. Chicago: The University of Chicago Press.

Kövecses, Z. 1986. Metaphors of Anger, Pride, and Love. John Benjamins Publishing Company: Amsterdam/Philadelfia.

Kövecses, Z. 2000. Metaphor and Emotion. Language, Culture and Body in Human Feeling. Cambridge: Cambridge University Press.

Lakoff, G. \& Johnson, M. 1980. Metaphors We Live By. Chicago: University of Chicago Press.

Lakoff, G. 1987. Women, Fire, and Dangerous Things: What Categories Reveal about the Mind. Chicago: University of Chicago Press.

Lakoff, G. 1999. Philosophy in the Flesh: The Embodied Mind and its Challenge to Western Thought. Basic books: New York.

Martín Mingorance, L. 1998. El Modelo Lexemático Funcional. Editora: Amalia Marín Rubiales. Editorial Universidad de Granada.

Martín Morillas, J.M.(en prensa). "The Semantics of Emotions: Towards an Integrated Approach".

Martín Morillas, J.M. 1997. "The cultural-cognitive model: a programatic application". Cuadernos de Filología Inglesa. Universidad de Murcia, 6/2:53-63. 
Martín Morillas, J.M. \& J.C. Pérez Rull. 1998. Semántica Cognitiva Intercultural. Granada: Método Ediciones.

Pérez Rull, J.C. 1998-99. "La conceptualización de la causa emocional del dolor y de la alegría. El esquema mental de la fuerza y su proyección metafórica". Revista Española de Lingüistica Aplicada. Vol. 13.

Pérez Rull, J.C. 2001. "La estructura conceptual y experiencial de las emociones. Modelos convencionales y fenomenógicos en el dolor emocional". Congreso AESLA. Alcalá de Henares. 357-362.

Rosch, E. 1978. "Principles of categorization». Cognition and Categorization, Eds. E. Rosch and L.L. Lloyd. Hillsdale, NJ: L. Earlbaum Associates. 27-48.

Wierzbicka, A. 1992. Semantics, Culture and Cognition: Universal Human Concepts in Culture-specific Configuration. Oxford University Press.

Wierzbicka, A. 1996. Semantics. Primes and Universals. Oxford University Press.

Wierzbicka, A. 1999. Emotion across Languages and Cultures. Diversity and Universals. Oxford University Press. 